\title{
Editorial: Diversifying Farming Systems for Adaptive Capacity
}

\author{
Margiana Petersen-Rockney ${ }^{1 *}$, Timothy Bowles ${ }^{1}$, Selena Ahmed ${ }^{2}$ and Patrick Baur ${ }^{3}$ \\ ${ }^{1}$ Department of Environmental Science, Policy, and Management, University of California, Berkeley, Berkeley, CA, \\ United States, ${ }^{2}$ Sustainable Food Systems Program, Department of Health and Human Development, Montana State \\ University, Bozeman, MT, United States, ${ }^{3}$ Sustainable Agriculture and Food Systems Program, Department of Fisheries, \\ Animal, and Veterinary Sciences, University of Rhode Island, Kingston, RI, United States
}

Keywords: diversified farming systems, adaptive capacity, multifunctionality, equity, sustainability

\section{Editorial on the Research Topic}

\section{Diversifying Farming Systems for Adaptive Capacity}

The COVID-19 pandemic underscores how novel shocks to agrifood systems compound the already dire global impacts of climate change, biodiversity loss, and food insecurity. Agrifood systems are not merely threatened by these interconnected crises-they drive them. In the pursuit of maximizing yield and profit, the ecologies, cultures, and markets that comprise our food systems have been made ever more uniform. Despite broad scientific agreement that agrifood systems must transform to mitigate climate change, conserve biodiversity and natural resources, and provide food and nutrition security in sustainable and equitable ways (Kremen and Merenlender, 2018; Shukla, 2019), much research remains agnostic toward how to do this. This collection of articles targets this gap by examining the social and ecological structures that shape adaptive capacity in farming systems and determine whether adaptive processes advance sustainability, resilience, and equity.

Agricultural adaptive capacity represents the extent to which farming systems can respond to climate change, biodiversity loss, and food insecurity in ways that not only preserve but holistically enhance their social-ecological functions. Petersen-Rockney et al. present a framework to analyze the relationship between farming system processes and adaptive capacity. By applying this framework to five diverse cases of agrifood system stressors, the authors find that adaptive capacity exhibits divergent qualities, such as heightened reliance on external inputs, in contrast to strengthening in-situ ecosystem service provisioning or place-based expertise, depending on the processes through which it emerges. Critically, the way we adapt to crises today shapes the range of possibilities for future adaptation. Returning to the former, usually simplified, state in response to crisis (i.e., a classic resilience perspective) may perpetuate crises. Instead, adaptations that diversify farming systems serve as long term investments from which robust in nimble adaptive capacity can emerge, helping social-ecological systems grow in more sustainable and equitable ways.

The 11 articles in this collection expand on these core insights, highlighting how adaptive capacity operates at various scales and levels, and emerges from diversification processes across ecological, social, and institutional dimensions of farming systems.

At the field and farm levels, diversification entails leveraging synergies and reducing tradeoffs among ecosystem, nutritional, and economic functions. Classically, farms can diversify through farming practices that strategically increase biodiversity across time and space to provision ecosystem services (Basche et al.; Stratton et al.). Diversification through practices like crop rotation can facilitate adaptive capacity to stressors like drought (Wauters et al.; Mortensen and Smith). For instance, Renwick et al. demonstrate how intercropping maize with a legume increases overall yield, improves nutritional quality, and increases adaptive capacity in the face of extreme weather-regardless of fertilizer availability. Renewed attention to crop maturity cycles offers another strategy to correct 
myopic focus on fast-maturing annual crops through reintroduction of long-duration (i.e., produce for several seasons), indeterminant (i.e., continuously producing), and perennial crops (Snapp).

Particularly among smallholders, the strategic (re)introduction of "neglected and underutilized crops" can balance on-farm nutritional needs with cash crop production, bolstering food sovereignty opportunities (van Zonneveld et al.). Q'eqchi' Maya smallholders who implemented ecological adaptation strategies, such as cover cropping or agroforestry, were better able to meet both nutritional and ecological goals compared to other farmers (Stratton et al.). Even conventional, highly industrialized farms can improve their ability to meet multiple goals simultaneously by, for example, incorporating lifecycle and habitat considerations for honeybees and other organisms (Durant and Ponisio).

Contributing authors also emphasized the importance of diversifying markets, values and goals, knowledge systems, farm business and management models, governing institutions and policies, and farmer demographics for improving adaptive capacity (Petersen-Rockney et al.). Multifunctional goals can help build structural diversification iteratively, aiding the emergence of adaptive capacity over time (Snapp). In contexts where multiple goals are prioritized together, coordinated for example through robust social networks and governance capacity, "total loss appears to provide a window of opportunity for reinventing agricultural systems" (Rodriguez-Cruz et al.). van Zonneveld et al. identify strengthening farmer-to-farmer networks, magnifying "lighthouse farmers" (e.g., custodians of traditional agroecological knowledge), and empowering women farmers as preconditions for on-farm diversification that safeguards household food security. Transformative potential also depends on the diversity of claims and claimants to resources like farmland (Calo). This point illuminates an implied corollary to van Zonneveld et al.'s argument that diversification begins with farmer goals: on-farm diversity is constrained by the diversity of functions that are sought from the land, and therefore on the diversity of people who have control over land use decisions.

While research articles in this collection find considerable evidence for the emergence of more broad and nimble adaptive capacity through diversifying processes, they also identify critical barriers to diversification. Mortensen and Smith emphasize the need to address factors that lock farmers into monocultures and narrow the range of adaptive possibilities. Snapp shows how maladaptive policies-such as India's Public Distribution System that "privileges" wheat and rice monocultures in the name of calorie-centric food security-lead to disabling lockin by pushing farmers into dependence on fossil fuels and chemical pest regulation. Basche et al. critique institutions that focus too narrowly on measuring singular agrifood production functions, calling instead for multifunctional metrics and investments that value a plurality of environmental and soil health co-benefits. Structural forces like land tenure regimes and crop incentive policies constrain individual farmers' agency (Rodriguez-Cruz et al.; Calo).

Contributing authors consistently agree that for broad and nimble adaptive capacity to emerge from farming systems, diversification efforts must embrace regionally specific messaging (Durant and Ponisio), site-specific implementation (Wauters et al.), and the unique contexts and goals of individual farmers (van Zonneveld et al.). Such multi-faceted and context-specific approaches can better identify synergies, as well as tradeoffs, between, for example, ecological and nutritional resilience functions (Stratton et al.).

As the articles in this collection demonstrate, diversifying farming systems recognizes a spectrum of socio-ecological practices that farmers and other agrifood workers can flexibly employ to increase adaptive capacity. Yet critical questions remain. Future work should assess diversifying approaches beyond the farm scale that can complement economic diversification of crop portfolios and biodiversification of genetic assets and ecosystem functions. Calo, for example, highlights the potential to diversify property relationships, while other authors nod toward market and insurance diversification (van Zonneveld et al.), heterogeneity of social networks and informal relationships (Rodriguez-Cruz et al.), diverse forms of knowledge and expertise (Petersen-Rockney et al.; van Zonneveld et al.), and new criteria and metrics for policy formation (Snapp; Basche et al.).

An important intervention this collection offers is distinguishing the state of being diversified or simplified from the processes of diversification and simplification. Obstacles to building socially just and environmentally sustainable adaptive capacity loom large. And low rates of adoption of diversification practices, such as basic legally required Best Management Practices among almond growers in California (Durant and Ponisio), may appear disheartening. However, focusing on processes helps illuminate pathways of opportunity. Instead of framing farmers as the "problem" in need of a "fix" through education, incentives, and regulation, we join Calo in suggesting a need to address the root causes of farming system vulnerability and inequity. We encourage ourselves, other scholars, and you, the reader, to engage in reflexive inquiry that always asks, adaptive capacity by whom and for whom?

\section{AUTHOR CONTRIBUTIONS}

MP-R suggested the Research Topic and outlined this editorial. MP-R, TB, SA, and PB met and discussed the topic and all contributions. All authors closely reviewed several articles in the collection, contributed points to the drafting of this text, edited this document, and approved its submission.

\section{FUNDING}

TB acknowledges USDA AFRI Grant \#2019-67019-29537 and NSF Coupled Natural Humans Systems Grant \#1824871.

\section{ACKNOWLEDGMENTS}

We would like to thank all the authors who contributed to this Research Topic. 


\section{REFERENCES}

Kremen, C., and Merenlender, A. M. (2018). Landscapes that work for biodiversity and people. Science 362:eaau6020. doi: 10.1126/science.aau6020

Shukla, P. R. (2019). IPCC, 2019: Climate Change and Land: An IPCC Special Report on Climate Change, Desertification, Land Degradation, Sustainable Land Management, Food Security, and Greenhouse Gas Fluxes in Terrestrial Ecosystems. Available online at: https://www.ipcc.ch/srccl/chapter/summaryfor-policymakers/ (accessed October 21, 2021).

Conflict of Interest: The authors declare that the research was conducted in the absence of any commercial or financial relationships that could be construed as a potential conflict of interest.
Publisher's Note: All claims expressed in this article are solely those of the authors and do not necessarily represent those of their affiliated organizations, or those of the publisher, the editors and the reviewers. Any product that may be evaluated in this article, or claim that may be made by its manufacturer, is not guaranteed or endorsed by the publisher.

Copyright $\odot 2021$ Petersen-Rockney, Bowles, Ahmed and Baur. This is an openaccess article distributed under the terms of the Creative Commons Attribution License (CC BY). The use, distribution or reproduction in other forums is permitted, provided the original author(s) and the copyright owner(s) are credited and that the original publication in this journal is cited, in accordance with accepted academic practice. No use, distribution or reproduction is permitted which does not comply with these terms. 